\title{
DETERMINATION OF COMPLEX STABILITIES WITH 1,10-PHENANTHROLINE- 5,6-DIONE AS LIGAND FOR THE COMPLEXATION OF SEVERAL TRANSITION METALLIC CATIONS USING CHEMOMETRICS METHODS
}

\author{
Naser Samadi* and Mina Salamati \\ Department of Chemistry, Faculty of Science, Urmia University, Urmia, Iran
}

(Received April 29, 2013; revised June 11, 2014)

\begin{abstract}
The traditional spectrophotometric determination of stability constants of complexation involves finding a wavelength at which only one of the components has absorbance without any spectroscopic interference of the other reaction components. But this method fails when there is not selective wavelength for at least one of the component. Multivariate data analysis involving soft and/or hard model analysis can be used in these cases. In the present work, firstly, multivariate curve resolustion-alternative least squares which is a model free analysis, was used to estimate the chemical model of data variation and then a robust multi-wavelength model-based method has been developed to determine the stability constants of formed complex(s) regardless to the spectra overlapping of the components. By applying such model, not only the stability constants of the formed complex(s) can be calculated, but also the pure spectra of all contributed components are extracted well. The complexations of 1,10-phenanthroline-5,6-dione ligand with several metalic cations [Cu(II), $\mathrm{Ni}(\mathrm{II}), \mathrm{Co}(\mathrm{II}), \mathrm{Zn}$ (II)] were studied using proposed multi-wavelength spectrophotometric method in non-aqueous solution at $25^{\circ} \mathrm{C}$. It was found that there is a 2:1 stoichiometry between ligand with studied metalic cations and also their stability constants were calculated.
\end{abstract}

KEY WORDS: Model free analysis, Model based analysis, Stability constant, Complexometric study, 1,10Phenanthroline-5,6-dione

\section{INTRODUCTION}

Coordination complexes are compounds and molecules formed between a metal ion and a ligand. The metal ions are essentially Lewis acids while the ligands are analogous to Lewis bases. Most often, the ligand has a lone pair of electrons that it donates to the empty d-orbitals in the metal. This forms a Lewis acid-base adduct.

Some applications have been recognized for this class of compounds [1]: catalysis, fabrication of high-performance materials, and use as molecular carriers in chemical or biochemical systems. In the recent years, some studies have dealt with the coordination properties of 1,10-phenanthroline-5,6-dione (PDON) in connection with its complexing ability towards both early and late transition metals $[2,3]$.

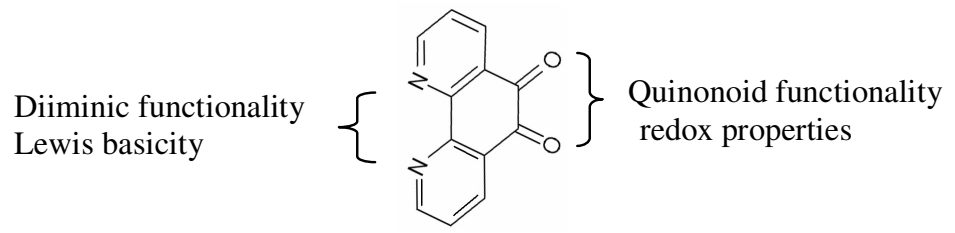

Scheme 1

1,10-Phenanthroline-5,6-dione, is a versatile molecule with applications in organic [4] and biological chemistry [5], and in the synthesis of materials showing interesting optical or

*Corresponding author. E-mail: samadi76@yahoo.com; na_samadi76@yahoo.com 
electrical properties [6]. This compound shows a peculiar reactivity due to the presence of two functionalities [2]: it is redox active due to the quinonoid function and it behaves as a Lewis base due to the presence of the two diiminic nitrogen atoms (Scheme 1). Calderazzo et al. [2], showed that PDON reacts with Lewis acids such as $\mathrm{TiCl}_{4}$ to give $\mathrm{N}, \mathrm{N}$-coordinated derivatives, and with a low-valent organometallic compound giving a redox reaction (Scheme 2). It has to be noted that when PDON coordinates through the nitrogen atoms, the entire complex, see I, may be used as 'quinone equivalent' in reactions with compounds containing metals in a low oxidation state. On the other hand, oxygen-bound complexes of 1,10- phenanthroline-5,6-dione such as II, may be used as a 'bipyridine equivalent' ligands in reactions with Lewis acids. In both cases, the result is the formation of complexes of higher nuclearity [2, 3]. Such ligands can be used in the production of chemical sensors because they show a significant change in the

color of the solution and maxima of the absorption band when they interact with transition metal ions.

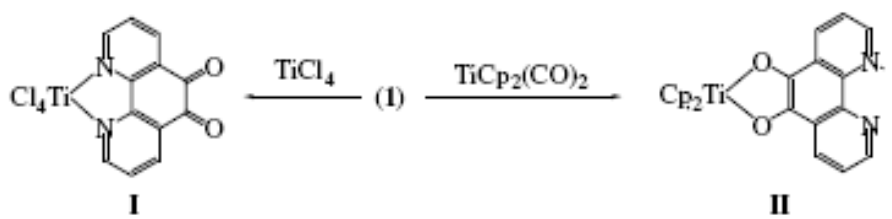

Scheme 2

Many experimental techniques are well known for studying the formation of complexes in solution. The most common experimental techniques for the determination of stability constants are potentiometry, conductometry, polarography, nuclear magnetic resonance spectroscopy, UV-Vis and fluorescence spectroscopy and calorimetry [7-13].

UV-Vis spectrophotometric methods have been frequently utilized to determine stability constants of complexes with large molar absorptivities because of its high sensitivity and easy interpretation and handling of the spectral data. On the other hand, the conventional spectrophotometric methods use a discrete number of wavelengths that frequently are not enough to furnish the necessary information to resolve a system with severe spectra overlapping. So, the direct spectrometric measurements show lack of specificity because the spectra are strongly overlapped. In such cases the chemometric techniques become an indispensable tool to overcome these problems [14-19].

There are two fundamentally different chemometric approaches for data analysis: modelbased analysis and model free analysis [20]. In model based analysis, the goal is to extract the basic parameters of the process using known chemical model. There are three distinct components in a model-based analysis; (a) the data, (b) the chemical model used to describe the chemical process and (c) the fitting of the parameters (e.g. equilibrium constants) that are pertinent to the model. On the other hand, in model free analysis there is no need to any previously known chemical model. The analysis is restricted to the determination of response curve, usually concentration profiles of the active species, and their spectral shapes [21].

In this work, the complexation behavior of 1,10-phenanthroline-5,6-dione with some metallic cations $[\mathrm{Cu}(\mathrm{II}), \mathrm{Ni}(\mathrm{II}), \mathrm{Co}(\mathrm{II}), \mathrm{Zn}(\mathrm{II})]$ have been studied using UV-Vis spectrophotometric method. Multivariate curve resolution-alternating least square (MCR-ALS) as a model-free analysis was used to extract approximate concentration profiles of components and get information about chemical model and then model based method was used to find model parameters, stability constant of complex in this case. 


\section{EXPERIMENTAL}

Reagents

All reagents and solvents were of commercial grade and used without further purification. The ligand 1,10-phenanthroline-5,6-dione (PDON) and all other reagents were purchased from Merck with the highest purity available.

Methods

All solutions were prepared using acetonitrile. The microliter amounts of concentrated metal cations solutions (e.g. $0-150 \mu \mathrm{L}$ from $10^{-4} \mathrm{M}$ ) were added to ligand solutions $\left(2 \mathrm{~mL}\right.$ from $10^{-5}$ M). The solutions were thermostated at $25^{\circ} \mathrm{C}$. An example for the change of the absorbance due to complex formation is given in Figure 1 that shows the complexation of $\mathrm{Ni}^{2+}$ by ligand PDON. Also Scheme 3 represents the possible structure of the $\mathrm{Cu}^{2+}, \mathrm{Co}^{2+}, \mathrm{Ni}^{2+}$ and $\mathrm{Zn}^{2+}$ with PDON.

All calculations in the computing process were performed in Matlab and Microsoft Excel for windows. A graphical interface of MCR-ALS [22], freely available on the web (http://www.ub.es/gesq/.mcr/mcr.htm), was used. Model based analysis was done using home written $\mathrm{m}$-file in Matlab.

\section{RESULTS AND DISCUSSION}

Treatment of the experimental data

The one step formation of a 2:1 complex between a ligand $\mathrm{L}$ and a cation $\mathrm{M}^{\mathrm{n}+}$ can be described as:

$$
\mathrm{M}^{\mathrm{n}+}+2 \mathrm{~L}^{\mathrm{p}-} \stackrel{\mathrm{k}_{\mathrm{f}}}{\longrightarrow} \mathrm{ML}_{2}^{\mathrm{n}-2 \mathrm{p}}
$$

And the corresponding stability constant is defined as:

$$
\mathrm{k}_{\mathrm{f}}=\frac{\left[\mathrm{ML}_{2}^{\mathrm{n}-2 \mathrm{p}}\right]}{\left[\mathrm{M}^{\mathrm{n}+}\right]\left[\mathrm{L}^{\mathrm{p}-}\right]^{2}}
$$

The mass balances for the total ligand $C_{\mathrm{L}}$ and total salt concentration $C_{\text {salt }}$ or total cation concentration $\mathrm{C}_{\mathrm{M}}$ are:

$$
\begin{aligned}
& \mathrm{c}_{\mathrm{L}}=\left[\mathrm{L}^{\mathrm{p}-}\right]+2\left[\mathrm{ML}_{2}^{\mathrm{n}-2 \mathrm{p}}\right] \\
& \text { and } \\
& \mathrm{c}_{\mathrm{M}}=\left[\mathrm{M}^{\mathrm{n}+}\right]+\left[\mathrm{ML}_{2}^{\mathrm{n}-2 \mathrm{p}}\right]
\end{aligned}
$$

The following equation can be derived using equations (1-4) with respect to ligand concentration:

$[\mathrm{L}]^{3}+\left(2 \mathrm{C}_{\mathrm{M}}-\mathrm{C}_{\mathrm{L}}\right) \mathrm{K}_{\mathrm{f}}[\mathrm{L}]^{2}+[\mathrm{L}]-\mathrm{C}_{\mathrm{L}}=0$

It should be noted that these equations could be derived as $\left[\mathrm{M}^{\mathrm{n}+}\right]$ or $\left[M L_{2}^{n-2 p}\right]$ concentrations. 
If there are selective wavelengths for any of mentioned components, ordinary method (fitting of results at a single wavelength) can be simply used for the estimation of $\mathrm{k}_{\mathrm{f}}$. This traditional method, as mentioned, fails when there is no any selective wavelength. Therefore ordinary method (using one-wavelength) cannot be used to accurate estimation of $\mathrm{k}_{\mathrm{f}}$. In such cases, multi-wavelength model-based analysis is a good alternative to estimate the formation constant of complex(s).

\section{Theory of model-based analysis}

For the multi-wavelength measurements of complexometric reaction in $n$ reaction concentration and $n \lambda$ wavelengths, $\mathbf{D}$ is decomposed into the product of $\mathbf{C}$, containing concentration profiles and $\mathbf{S}$ of absorption spectra (absorption data are governed by Beer-Lambert's law and the measurements are well described by a matrix equation):

$\mathbf{D}=\mathbf{C S}^{\mathrm{T}}+\mathbf{E}$

where $\mathbf{D}$ is a matrix, the rows of which are formed by the absorption spectra measured as a function of the progress of the process. The columns of $\mathbf{D}$ are the absorption traces measured at different wavelengths. According to Beer-Lambert's law, this matrix can be decomposed into the product of a matrix $\mathbf{C}$ containing the concentration profiles of the absorbing species at different mole ratio and a matrix $\mathbf{S}$ containing their molar absorptivities. The matrix $\mathbf{E}$ is a collection of the residuals, the difference between the measurement $\mathbf{D}$ and its calculated representation $\mathbf{C S}^{\mathrm{T}}$.

Nonlinear least squares hard-modeling consists of finding set of parameters for which the sum over all the squares, ssq, over all the elements of the error matrix, $\mathbf{E}$, is minimal [26]. This crucial sum is a function of the measurement, $\mathbf{D}$, the pre-defined model and the parameters:

$\mathrm{ssq}=\sum \mathrm{E}_{\mathrm{i}, \mathrm{j}}^{2}=\mathrm{f}(\mathbf{D}, \bmod$ el, parameters $)$

The "roots" command of MATLAB was applied for solving the $3^{\text {rd }}$ order equation (Eq. 5) in order to estimate the concentration profile of $\mathrm{L}$ and establish the matrix $\mathbf{C}$ (concentration profiles of all involved compounds) by initial estimation of equilibrium constants. It is of utmost importance to recognize that the molar absorptivities are linear parameters, which can be effectively eliminated from the list of parameters that need to be fitted iteratively. At any stage in the process, the best linear parameters are explicitly calculated as $\mathbf{S}^{\mathrm{T}}=\mathbf{C}^{+} \mathbf{D}$, where $\mathbf{C}^{+}$is the pseudo-inverse of the matrix $\mathbf{C}$ [20]. Thus, for a given model, the sum of squares can be defined as a function of only the nonlinear parameters (e.g. the equilibrium constant(s) in this case), and these nonlinear parameters, which define $\mathbf{C}$, are the only ones that need to be fitted in an iterative process. Summarizing, given the model and the measurement D, Eq. (7) can be written as

$\operatorname{ssq}=\mathbf{f}(\mathbf{k})=\mathbf{D}-\mathrm{CS}^{\mathrm{T}}=\mathrm{D}-\mathrm{CC}^{+} \mathrm{D}$

where $\mathbf{k}$ is the nonlinear parameter(s), as defined by the chosen model. Any method that performs this minimization task can be applied. In this work, the Newton-Gauss-LevenbergMarquardt algorithm (NGLM) was applied because it is fast, robust and additionally delivers basic statistical analysis of the resulting parameters [20].

The first step in hard-modeling processes is the establishment of the correct model. In other words, model-fitting is much easier than mode-finding [23]. This is generally the most difficult task and requires the knowledge and experience of the researcher. For this purpose, model free 
approach such as MCR-ALS as very useful method was used to estimate the right model in this work.

Theory of model-free analysis

The ALS optimization algorithm for curve resolution has been described in detail elsewhere [21, $22,24]$ and is only briefly introduced here.

The bilinear model assumed by MCR methods is analogous to the generalized LambertBeer's law, where the individual responses of each component are additive. In matrix form, this bilinear model is expressed as Eq. 6. The iterative ALS procedure is initialized using an initial estimation of the spectral or concentration profiles for each intervening species. If the initial estimations were the spectra, the unconstrained least-squares solution for the concentration profiles can be calculated from the Eq. 9:

$\mathbf{C}=\mathbf{D}\left(\mathbf{S}^{\mathrm{T}}\right)^{+}$

where " + " denotes the pseudo-inverse of the matrix. After calculation of C using the Eq. (9), $\mathbf{C}_{\text {new }}$ is achieved by implementation of some constraints on $\mathbf{C}$. Then, $\mathbf{S}_{\text {new }}{ }^{\mathrm{T}}$ is calculated using Eq. 10:

$\mathbf{S}_{\text {new }}{ }^{\mathrm{T}}=\mathbf{C}_{\text {new }}{ }^{+} \mathbf{D}$

These calculations continue until convergence is achieved. At each cycle, a new estimation of $\mathbf{S}^{\mathrm{T}}$ and $\mathbf{C}$ is calculated by solving alternatively the two above least-squares matrix equations. At every iteration, constraints are applied to drive the optimization process towards a final solution [25].

There are infinite numbers of solutions for $\mathbf{C}$ and $\mathbf{S}$ in Eq. 6. This infinite number of possible solutions can usually be considerably reduced by means of constraints derived from the physical nature and previous knowledge of the system under study. A constraint can be defined as any mathematical or chemical property fulfilled by the whole system or by some of its pure contributions [26]. For instance, only positive values for the concentrations of the chemical components in the mixture have physical meaning. In many spectroscopic techniques, only positive values are allowed in the spectra; concentration profiles are often unimodal; and closure or mass balance equations should be fulfilled for reaction-based systems. Also, the implementation of a physicochemical model in the resolution process can help to decrease the ambiguity of the related profiles quite considerably. MCR-ALS has been used for different purposes [27-31], in this work it was used for model finding.

Analysis of experimental data

Figure 1 represents the complexometric spectra for the complexation of $\mathrm{Ni}^{2+}$ by ligand PDON where mole ratio of $\left[\mathrm{Ni}^{2+}\right] /[\mathrm{PDON}]$ is in the range of $0.0-2.5$. As can be seen, the absorbance about $252 \mathrm{~nm}$ decreases by addition of $\mathrm{Ni}^{2+}$. Also an increase in absorbance can be seen at about $270-330 \mathrm{~nm}$. An isobestic point can be found at $268 \mathrm{~nm}$. These results admit that the system may obey a one step complexation reaction between $\mathrm{Ni}^{2+}$ and PDON. In order to validate this result, singular values decomposition, as a powerful mathematical tool, was used. Singular value decomposition (SVD) can be looked at from three mutually compatible points of view. On the one hand, we can see it as a method for transforming correlated variables into a set of uncorrelated ones that better expose the various relationships among the original data items. At the same time, SVD is a method for identifying and ordering the dimensions along which data points exhibit the most variation. Briefly SVD can be seen as a method for data reduction and 
the singular values can be used simply to find out the number of significant components in a data matrix.

Singular value decomposition (SVD) showed that there are 3 components in the investigated wavelength range $210-610 \mathrm{~nm}$ (Figure 2). Whereas $\mathrm{Ni}^{2+}$ (solvated cation) and PDON as a ligand (L) are spectroscopic active in this wavelength range so the third component can be attributed to the formed complex. All these results admit that there is a one step complexation reaction between $\mathrm{Ni}^{2+}$ and PDON.

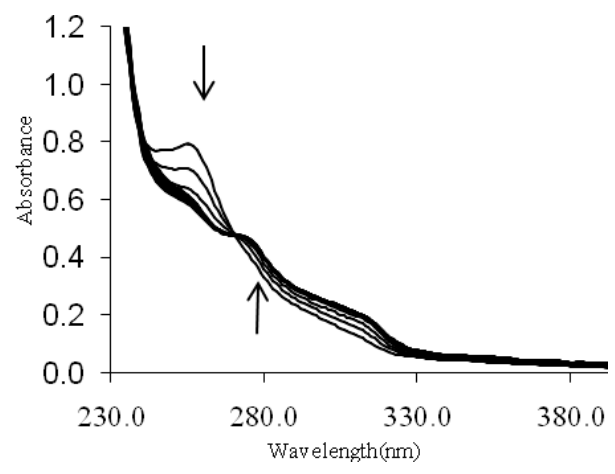

Figure 1. Changes in UV-Vis absorption spectra of PDON in acetonitrile solvent $\left(3.8 \times 10^{-5} \mathrm{~mol}\right.$ $\mathrm{L}^{-1}$ ) by adding $\mathrm{Ni}^{2+}$. Arrows indicate a change in the absorbance with increase in the concentration of $\mathrm{Ni}^{2+}$ from a $\left[\mathrm{Ni}^{2+}\right] /[\mathrm{PDON}]$ mole ratio of 0.0 to 2.5 .

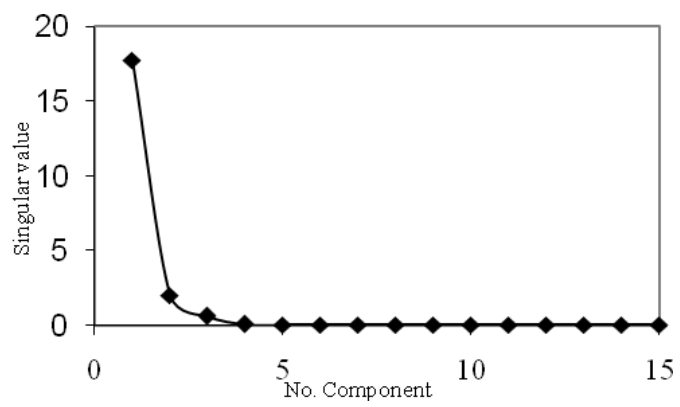

Figure 2. Consecutive singular values of singular value decomposition of $\mathrm{Ni}^{2+}$-PDON complexometric data matrix as a function of number of components.

Because of serious spectral overlapping among metal cation, ligand and formed complex, it is not possible to use traditional mole ratio or Job's plot to get information about the stoichiometry and stability constant of complex of $\mathrm{Ni}^{2+}$ with PDON. For this reason, MCR-ALS, as a model free analysis, was used to resolve the data matrix and extract the concentration profiles of three components. Since the spectra for reactants were available, so the optimization step of MCR-ALS was initiated using the spectra of the individual components of $\mathrm{Ni}^{2+}$ and PDON and last spectrum of the data (as estimation of formed complex spectrum).

A series of constraints was applied in an attempt to improve the optimization and to restrict the number of possible solutions: (a) the pure spectra of each component must be non-negative; (b) the concentration profiles of each component must be non-negative; (c) concentration 
profiles of each component only have one maximum known as unimodality constraint. After getting convergency in ALS optimization, concentration profiles and pure spectra were obtained (not shown here). The obtained concentration profile of formed complex leveled off about 0.5 values of $\left[\mathrm{Ni}^{2+}\right] /[\mathrm{PDON}]$. This means that there is a $2: 1$ stoichiometry between ligand $\mathrm{L}$ and cation $\mathrm{Ni}^{2+}$. Therefore absorbing components in this system could be assumed as $\mathrm{L}^{2} \mathrm{NiL}_{2}$ and $\mathrm{Ni}^{2+}$ (solvated cation).

Model based analysis was then applied for accurate determination of $\mathrm{K}_{\mathrm{f}}$ as well as resolving the system and acquiring the pure spectra. Initial estimate of $\mathrm{K}_{\mathrm{f}}$ was used to construct the Eq. 5 for any mole ratio and then solved them using "root" command of MATLAB, finally NGLM algorithm was used to find optimum value of $\mathrm{K}_{\mathrm{f}}$. After finding optimum value of $\mathrm{K}_{\mathrm{f}}$, concentration profiles and also pure spectra of components can be obtained. The results are shown in Figure 3. The equilibrium constant of $\mathrm{NiL}_{2}$ complex was calculated to be $10^{9.6}-10^{9.7}$ using different initial estimates.

a

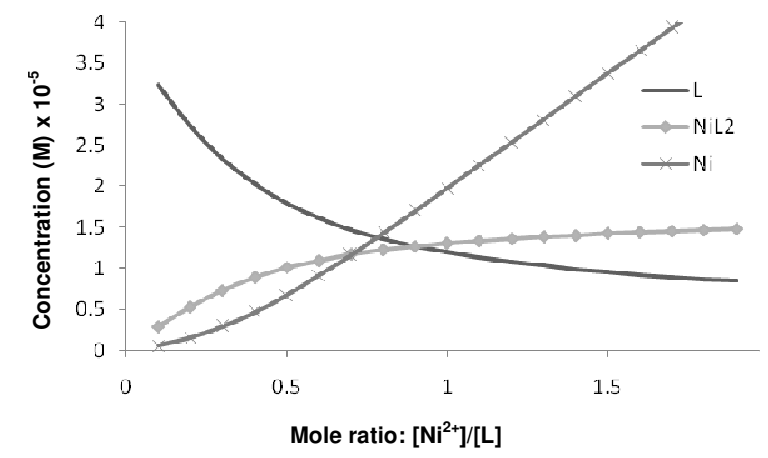

b

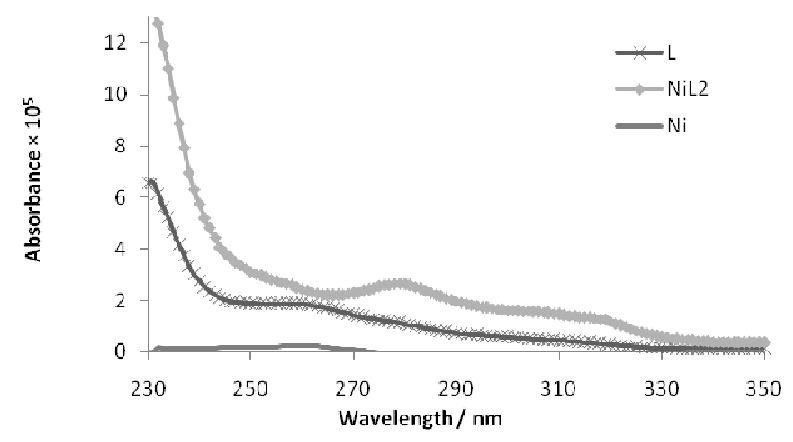

Figure 3. Results of model based analysis of complexation of $\mathrm{Ni}^{2+}$ with PDON in acetonitrile (a) resolved concentration profile and (b) resolve spectra.

The same studies were done for the complexation of $\mathrm{Cu}^{2+}, \mathrm{Co}^{2+}$ and $\mathrm{Zn}^{2+}$ with 1,10 phenanthroline-5,6-dione in acetonitrile. Figure 4 represents the complexometric spectra of PDON with $\mathrm{Cu}^{2+}$. 


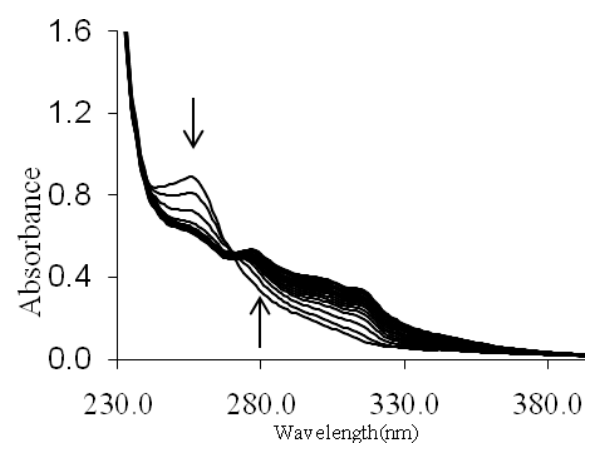

Figure 4. Changes in UV-Vis absorption spectra of PDON in acetonitrile solvent $\left(3.8 \times 10^{-5} \mathrm{~mol}\right.$ $\mathrm{L}^{-1}$ ). Arrows indicate a change in the absorbance with increase in the concentration of $\mathrm{Cu}^{2+}$ from a $\left[\mathrm{Cu}^{2+}\right] /[\mathrm{PDON}]$ mole ratio of 0.0 to 2.5 .

The number of significant component was estimated using singular value decomposition algorithm. Figure 5 shows that there are 3 components in this matrix. The stoichiometry between ligand $\mathrm{L}$ and cation $\mathrm{Cu}^{2+}$ was found 2:1 using model free analysis. The equilibrium constant of $\mathrm{CuL}_{2}$ complex was calculated to be $10^{10.4}$ using model based analysis. The resulted concentration profiles and the spectra are shown on Figure 6.

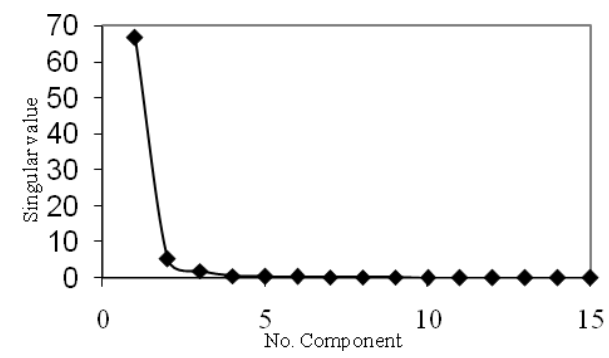

Figure 5. Consecutive singular values of singular value decomposition of $\mathrm{Cu}^{2+}$-PDON complexometric data matrix as a function of number of components. 


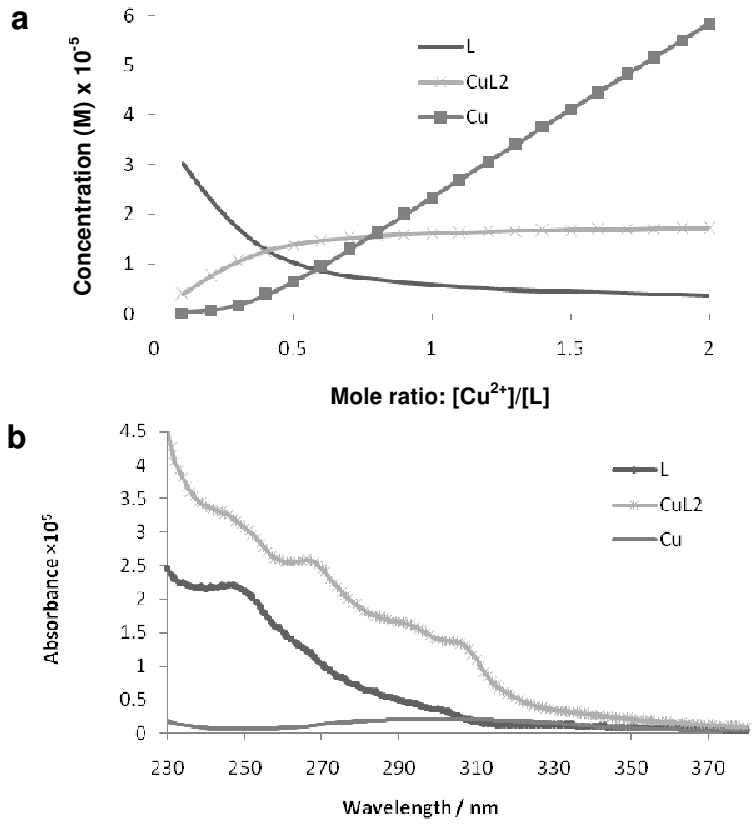

Figure 6. Results of model based analysis of complexation of $\mathrm{Cu}^{2+}$ with PDON in acetonitrile (a) resolved concentration profile and (b) resolve spectra.

The proposed method was used to resolve the complexation data matrix of $\mathrm{Zn}^{2+}$ and $\mathrm{Co}^{2+}$ with PDON. The equilibrium constants of $\mathrm{ZnL}_{2}$ and $\mathrm{CoL}_{2}$ complexes were also calculated as $10^{8.37}$ and $10^{7.89}$, respectively.

\section{CONCLUSION}

In this work, model free and model based methods were used to analyze spectrophotometric data of complexation of metallic cations [Ni(II), $\mathrm{Co}(\mathrm{II}), \mathrm{Zn}(\mathrm{II})$ and $\mathrm{Cu}$ (II)] with an insoluble ligand named 1,10-phenanthroline-5,6-dione. The stability constants of these metal complexes were calculated and also concentration profiles and pure spectra of components were obtained. The results showed that these cations form stable complexes with this ligand. These results suggest that this ligand could be used as a new active emissive probe to detect these cations especially $\mathrm{Cu}(\mathrm{II})$.

\section{REFERENCES}

1. Newkome, G.R.; He, E.; Moorefield, C.N. Chem. Rev. 1999, 99, 1689.

2. Calderazzo, F.; Marchetti, F.; Pampaloni, G.; Passarelli, V. J. Chem. Soc., Dalton Trans. 1999, 4389.

3. Paw, W.; Eisenberg, R. Inorg. Chem. 1997, 36, 2287.

4. López, R.B.; Loeb, B.L.; Boussie, T.; Meyer, T.J. Tetrahedron Lett. 1996, 37, 5437.

5. Shabir, G.A.; Forrow, N.J. J. Pharm. Biomed. Anal. 2003, 33, 219.

6. Tobalina, F.; Pariente, F.; Hernández, L.; Abruña, H.D.; Lorenzo, E. Anal. Chim. Acta 1999, $395,17$. 
7. El-Sherif, A.A. J. Solution Chem. 2012, 41, 392.

8. Shoukry, A.A.; Shoukry, M.M. Spectrochim. Acta, Part A 2008, 70, 686.

9. Martins, J.G.; Gameiro, P.; Barros, M.T.; Soares, H.M.V.M. J. Chem. Eng. Data 2010, 55, 3410.

10. Arancibia, J.A.; Escandar, G.M. Analyst 1999, 124, 1833.

11. Esteban, M.; Ariño, C.; Díaz-Cruz, J.M.; Díaz-Cruz, M.S.; Tauler, R. Trends Anal. Chem. 2000, 19, 49.

12. Almandoz, M.C.; Dávila, Y.A.; Sancho, M.I.; Gasull, E.I.; Blanco, S.E. Spectrochim. Acta, Part A 2010, 77, 51.

13. Shamsipur, M.; Samadi, N.; Irandoust, M. Pol. J. Chem. 2004, 78, 1979.

14. Shamsipur, M.; Ghavami, R.; Hemmateenejad, B.; Sharghi, H.; Alizadeh, K.; Niknam, K. Pol. J. Chem. 2008, 82, 1621.

15. Shamsipur, M.; Maddah, B.; Hemmateenejad, B.; Rouhani, S.; Haghbeen, K.; Alizadeh, K. Spectrochim. Acta, Part A 2008, 70, 1.

16. Afkhami, A.; Khalafi, L. J. Chin. Chem. Soc. 2007, 54, 957.

17. Afkhami, A.; Madrakian, T.; Keypour, H.; Soltanbeygi, S.; Khajavi, F.; Rezaeivala, M. J. Mol. Struct. 2011, 985, 86.

18. Ghasemi, J.; Niazi, A.; Maeder, M. J. Braz. Chem. Soc. 2007, 18, 267.

19. Bahram, M.; Pesyan, N.N.; Naseri, A.; Tasbihforosh, M. Turk. J. Chem. 2011, 35, 255.

20. Norman, S.; Maeder, M. Crit. Rev. Anal. Chem. 2006, 36, 199.

21. Juan, A. De.; Tauler, R. Crit. Rev. Anal. Chem. 2006, 36, 163.

22. Jaumot, J.; Gargallo, R.; Juan, A. De.; Tauler, R. Chemom. Intell. Lab. Syst. 2005, 76, 101.

23. Maeder, M.; Neuhold, Y.M. Practical Data Analysis in Chemistry, 1st ed., Elsevier: Amsterdam; 2007.

24. Garrido, M.; Rius, F.X.; Larrechi, M.S. Anal. Bioanal. Chem. 2008, 390, 2059.

25. Goicoechea, H.C.; Olivieri, A.C.; Tauler, R. Analyst 2010, 135, 636.

26. DeJuan, A.; VanderHeyden, Y.; Tauler, R.; Massart, D.L. Anal. Chim. Acta 1997, 346, 307.

27. Khataee, A.R.; Safarpour, M.; Naseri, A.; Zarei, M. J. Electroanal. Chem. 2012, 672, 53.

28. Naseri, A.; Abdollahi, H. Anal. Methods 2011, 3, 429.

29. Naseri, A.; Ayadi-Anzabi, H. Anal. Methods 2012, 4, 153.

30. Naseri, A.; Bahram, M.; Mabhooti, M. J. Braz. Chem. Soc. 2011, 22, 2206.

31. Sorouraddin, M.H.; Amini, K.; Naseri, A.; Asgari, D.; Rashidi, M.R. Bioprocess Biosyst. Eng. 2011, 34, 173. 